\title{
COAL AND COAL CONSTITUENT STUDIES \\ BY ADVANCED EMR TECHNIQUES
}

\author{
Semi-Annual Technical Report \\ Reporting Period: 03/01/1996 - 08/31/1997
}

Authors: R. Linn Belford, Robert B. Clarkson, Boris Odintsov, Paul J. Ceroke

Report Issue Date: 09/30/1997

DE-FG22-96PC96205

University of Illinois at Urbana-Champaign

Department of Chemistry - Box 18.6 CLSL - MC712

$600 \mathrm{~S}$. Mathews

Urbana, IL 61801 
Semiannual Technical Report to DOE - PETC - Grant \# DE-FG22-96PC96205 9/1/96-2/28/97

\title{
COAL AND COAL CONSTITUENTS STUDIES BY ADVANCED EMR TECHNIQUES
}

\author{
by R. Linn Belford, Robert B Clarkson, Boris M. Odintsov, and Paul J. Ceroke \\ University of Illinois at Urbana-Champaign
}

\begin{abstract}
DISCLAIMER
This report was prepared as an account of work sponsored by an agency of the United States Government. Neither the United States Government nor any agency thereof, nor any of their employees, makes any warranty, express or implied, or assumes any legal liability or responsibility for the accuracy, completeness, or usefulness of any information, apparatus, product, or process disclosed, or represents that its use would not infringe privately owned rights. Reference herein to any specific commercial product, process, or service by trade name, trademark, manufacturer, or otherwise does not necessarily constitute or imply its endorsement, recommendation, or favoring by the United States Government or any agency thereof. The views and opinions of authors expressed herein do not necessarily state or reflect those of the United States Government or any agency thereof.
\end{abstract}

\begin{abstract}
Advanced electronic magnetic resonance (EMR) methods are used to examine properties of coals, chars, and molecular species related to constituents of coal. During this grant period, progress was made on a high frequency EMR system particularly appropriate for such studies and on low-frequency dynamic nuclear polarization (DNP) to examine the interaction between fluids such as water and the surface of suspended char particles.
\end{abstract}

\section{TABLE OF CONTENTS}

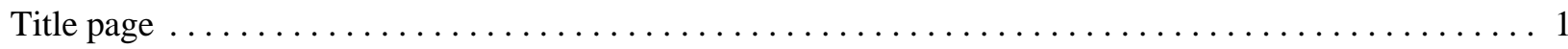

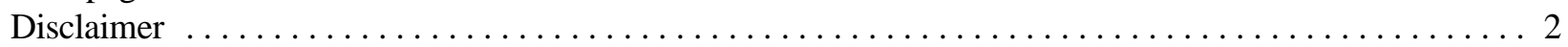

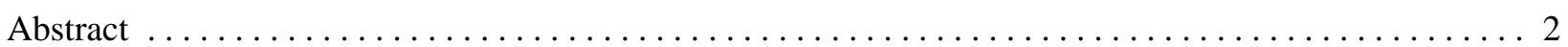

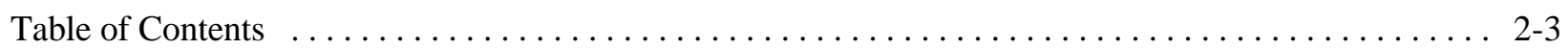

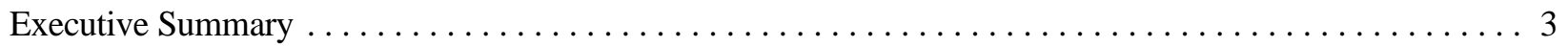

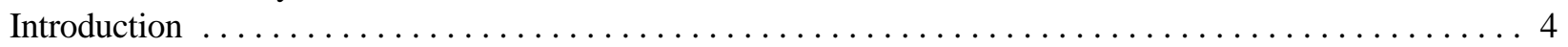

Measurements ........................................... $4-6$

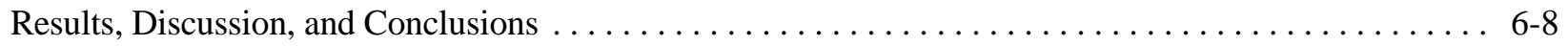

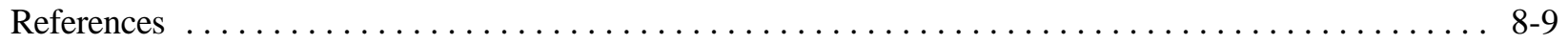

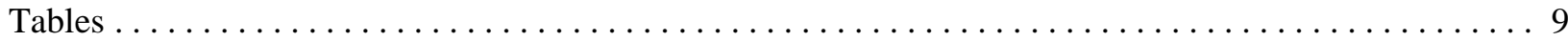

1. DNP Data

2. Self-diffusion coefficients and NMR relaxation data) 


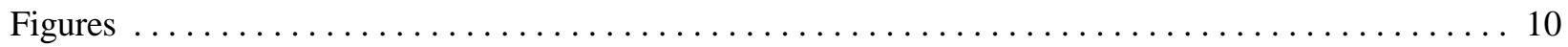

1. Temperature dependence of DNP enhancements of water protons in aqueous suspensions of chars made from: a) hardwood and b) fructose. $B_{1 S}=0.1 \mathrm{G}, \tau_{\mathrm{p}}=0.5 \mathrm{~s}, \tau_{\mathrm{d}}=4 \mathrm{~ms}$.

2. Typical raw PFG-NMR data. Plot of $\ln \left(\mathrm{A}_{\mathrm{g}} / \mathrm{A}_{\mathrm{o}}\right)$ vs. $\mathrm{k}$ for two chars saturated with water. The squares, obtained from water diffusing in hardwood char suspensions, clearly illustrate the effects of restriction on the PFG-NMR diffusion experiment. The circles, taken from water in fructose suspensions under the same experimental conditions, represent the curve for unrestricted diffusion.

3. Schematic illustrating the effect of restricting boundaries on water diffusion and relaxation measurements in char suspensions.

4. Dependence of leakage factor $f$ on the sticking times of $\tau^{\mathrm{p}}$ and $\tau^{\mathrm{s}}$ for fructose char suspensions.

\section{EXECUTIVE SUMMARY}

The main advanced magnetic resonance methods employed in the second six months of this project were (1) W-band $(100 \mathrm{GHz})$ electronic magnetic resonance (EMR, EPR, or ESR) spectroscopy to provide an analytical tool to discriminate among very similar chemical species that are constituents of coals and closely related materials such as chars, and (2) very low frequency pulsed dynamic nuclear polarization (DNP) spectroscopy for the study of the interfaces between coal-like particles and the water medium in which they are suspended. Considerable progress on enhancing the W-band spectrometer will be described in a future report.

This report focuses on the DNP work. The previous Technical Report described the first observation of solid-liquid electron spin density transfer in an aqueous system consisting of carbonaceous particles - hardwood chars suspended in water - by a proton pulsed DNP technique at low magnetic field. Proton DNP enhancement was seen for aqueous suspensions of several newly synthesized types of chars, where both positive and negative Overhauser effects were detected in different samples [1-3].

Heterogeneous ${ }^{1} \mathrm{H}$ Dynamic Nuclear Polarization (DNP) at low magnetic field in several newly synthesized chars suspended in water is experimentally studied by several advanced magnetic resonance techniques. By using Fourier Transform Pulsed-Field-Gradient spin-echo NMR spectroscopy several self-diffusion coefficients have been obtained in aqueous char suspensions corresponding to different mobility of water molecules in porous structure. Proton spin-lattice relaxation data generally confirm the results of molecular diffusion measurements. On the basis of the Torrey model, the influence of "cage effect" on DNP enhancement in porous media is discussed. It is concluded that the short-range nuclear-electronic interactions in pore space have the dominant effect on DNP enhancement in char suspensions.

In general, the unpaired electron spins used to dynamically polarize the nuclei are distributed uniformly throughout the bulk of the sample. In some cases, DNP can be produced in a liquid by pumping with microwave radiation in resonance with paramagnetic centers near a solid-liquid interface [4]. There are only a few reported experimental studies of solid-liquid DNP transfer between sublevels in magnetic spin systems consisting of species with different gyromagnetic ratios [5-6]. Most of these have described the dominant "solid-state" DNP effect due to pure dipole-dipole intermolecular interactions, such as those observed between free radical labels immobilized on a surface and solvent protons [5], in contrast to the current work. 


\section{INTRODUCTION}

The interactions of water in coal are important factors in all aspects of coal cleaning and utilization. It has long been suspected that water adsorbed in the pores of the coal matrix effects the EPR spectral line shapes seen by advanced Electron Magnetic Resonance (EMR) methods. Recently, we developed a very low-field pulsed dynamic nuclear polarization (DNP) spectrometer capable of observing the interactions of water with the surfaces of carbonaceous solids. In our first report, we described our first experiments making use of this novel DNP instrument, and outlined some of the new insights which this approach can provide for coal research. The first observation of solid-liquid electron spin density transfer by the contact Overhauser mechanism $[7,8]$ seen in a system consisting of hardwood chars suspended in water was reported. Proton Dynamic Nuclear Polarization (DNP) enhancement was investigated in aqueous suspensions of several types of chars, where both positive and negative Overhauser effects were detected in different samples. The positive DNP effect was strong evidence for the presence of an electronnuclear scalar interaction at solid-liquid interface. To date, only a few studies have been published of solid-liquid energy transfer between sublevels in magnetic spin systems consisting of species with different gyromagnetic ratios $[9,10]$. All of these have described the dominant "solid-state" DNP effect due to pure dipole-dipole (through space) intermolecular interactions, such as those observed between free radical labels immobilized on a silica gel surface and solvent protons [9].

Contact DNP in solutions of paramagnetic ions is seldom observed for protons because of the very small contact hyperfine coupling that usually exists between unpaired electrons in a solute molecule and hydrogen atoms in solvent molecules. Until now, contact DNP for water protons was only observed in aqueous solutions of $\mathrm{Mn}^{2+}$ and Wurster's blue perchlorate [11,12]. The scalar coupling we have seen in aqueous hardwood char suspensions demonstrates for the first time that there is electron delocalization from paramagnetic centers on the surface to water protons in the solvent, since the contact interaction demands that the electronic wave function at the nucleus be non-zero. The positive DNP effect was accompanied by a significant difference between spin-lattice and spin-spin relaxation times of water protons. The proton DNP enhancement observed in aqueous char suspensions was strongly temperature dependent. In contrast to ordinary free radical solutions, increasing scalar polarization with temperature was observed. These results suggest the possibility of exchange processes at the solid-liquid interface. For several hardwood chars the sign of the water proton DNP enhancement changed from negative to positive, indicating a change in the relative contributions from scalar and dipole-dipole nuclear-electron coupling.

\section{MEASUREMENTS}

An understanding of molecular motion and spin dynamics in porous structure is of central importance to research concerning heterogeneous catalysis, fluid penetration of engineering plastics and ceramics, and biological perfusion. Due to the confining influence of pore boundaries on the molecular motion, this motion contains a signature characteristic of pore 
morphology. Studies of molecular diffusion and relaxation processes in paramagnetic porous materials provide valuable information about surface relaxivity and spin dynamics on solidliquid interfaces [4-5].

Over the past two decades, magnetic resonance methods have been used extensively in studies of paramagnetic centers in carbonaceous solids as well as in studies of the surface diffusion of solvents adsorbed on charcoals [13-19]. In spite of significant progress in these studies, the electronic structure of char paramagnetic centers and the process of free radical formation during carbonization, as well as the nature of hyperfine interactions on the surface of chars, are not yet fully understood. Here is described the results of the heterogeneous DNP effect study in chars suspended in water by using several advanced magnetic resonance techniques, enumerated as follows:

\section{Pulsed 1H DNP:}

The measurements of DNP enhancement of water protons in char suspensions were carried out under the following conditions: $v_{\mathrm{I}}=\omega_{\mathrm{I}} / 2 \pi=0.5 \mathrm{MHz}, \mathrm{B}_{\mathrm{o}}=117.5 \mathrm{G}$; the electron resonance frequency was $v_{\mathrm{S}}=328.8 \mathrm{MHz}$. Details of the experimental apparatus were described in the previous report and elsewhere $[1,2,3,20]$.

\section{Pulsed-Field-Gradient (PFG) spin-echo NMR spectroscopy.}

PFG NMR has attracted renewed attention as a probe of microscopic but volumetrically averaged properties of materials [21-24]. This method is one of the best tools for measuring time-dependent diffusion coefficients [22]. The measurement is nondestructive and does not involve the introduction of chemical or isotopic tracers [21]. The quantitative relationship between spin-echo amplitude attenuation and self-diffusion coefficient $\mathrm{D}$ is given by equation

$$
\ln \left(\mathrm{A}_{\mathrm{G}} / \mathrm{A}_{\mathrm{o}}\right)=\left[-\gamma^{2} \mathrm{D} \delta^{2} g^{2}(\tau-\delta / 3)\right]
$$

where $\gamma$ is the gyromagnetic ratio of the nucleus, $\delta$ is the duration of the pulsed field gradient, $\mathrm{A}_{\mathrm{G}}$ is the spin-echo signal amplitude and $\mathrm{A}_{\mathrm{o}}$ is the amplitude with no gradients. Multi-component mixture analysis allows one to account for the spectra of self diffusion coefficients of individual components in a dispersion.

The measurements of self-diffusion coefficients of water molecules in char suspensions were performed at room temperature with a modified version of a high-resolution NMR spectrometer (BS-567A), operated at a Larmor ${ }^{1} \mathrm{H}$ frequency of $100 \mathrm{MHz}$ and equipped with a Fourier transform accessory. The spectrometer was modified with two self-shielded gradient Helmholtz coils to provide a maximum gradient strength of $50 \mathrm{G} / \mathrm{cm}$. The standard relaxation Hahn pulsed sequence program was modified to introduce the gradient pulses as well as a delay $\tau$ between the end of the second RF pulse and the start of data accumulation, the delay being equal to the time between two pulses. Experiments were performed by varying the gradient strength $g$ and keeping the gradient width $\delta$ and all other timing parameters constant.

\section{NMR relaxometry.}

Spin-lattice relaxation times $\mathrm{T}_{1}{ }^{\mathrm{i}}$ of water protons in char suspensions have been measured 
Semiannual Technical Report to DOE - PETC - Grant \# DE-FG22-96PC96205 9/1/96-2/28/97

by an NMR relaxometer (NMR-07PC). Special software has been created to account for several NMR relaxation times in a system in which the relaxation (echo-decay) curve was described as a sum of several components, $\mathrm{A}(\mathrm{t}) / \mathrm{A}(0)=\sum \mathrm{P}_{\mathrm{i}} \mathrm{F}_{\mathrm{i}}(\mathrm{t})$, with corresponding weighting coefficients, $\mathrm{P}_{\mathrm{i}}$. An exponential form was assigned to each component $F_{i}(t)=\exp \left(-t / T_{1}\right)$. Experiments were carried out at room temperature at the proton resonance frequency, $6 \mathrm{MHz}$. The pulse sequence $\left(90^{\circ}-\tau\right.$ $\left.90^{\circ}-\tau-180^{\circ}\right)$ was used.

\section{Samples.}

Samples of chars used in this work were produced by a thermal treatment of carbonaceous materials obtained from various kinds of woods (e.g. hardwoods, softwoods), as well as from starch and fructose, by charring under an $\mathrm{H}_{2}$ flow. Preparation techniques included digitally programed temperature profiles, ball milling, and size separation by micro-sieving. A particle size of about $450 \mu \mathrm{m}$ was used in these experiments. The samples were suspended in water, and then bubbled with pure helium for $15 \mathrm{~min}$. to reduce the concentration of dissolved oxygen in the solvent. The char suspensions were prepared at a concentration $0.64 \mathrm{~g} / \mathrm{cm}^{3}$ of water.

Experimental details of char synthesis were described in the previous report and elsewhere $[12,25]$.

\section{RESULTS, DISCUSSION, and CONCLUSIONS}

In this study, the proton DNP enhancement of water molecules was observed in aqueous suspensions of several newly synthesized types of chars, where both positive and negative Overhauser effects were detected in different samples. Table 1 shows DNP parameters and proton spin-lattice relaxation times $\mathrm{T}_{1}$ for chars suspended in water with different nuclear-electron interactions.

A strong temperature dependence of the DNP enhancement was observed in aqueous suspensions of hardwood chars (Fig.1-a). The increase in scalar polarization with temperature suggests the possibility of exchange processes at the solid-liquid interface. We constructed a model of molecular migration at the solid-liquid interface [11] to account for these observations. In contrast to hardwood chars, the negative DNP enhancement in fructose char suspensions was practically temperature independent (Fig.1-b), suggesting that the frequency dispersion [8] is negligible in these systems and that the "white spectrum" condition was realized in the frequency region investigated. Temperature independence of DNP enhancement in fructose char suspensions indicates also dominance of the dipole-dipole type of hyperfine interaction at solidliquid interface, because the change in temperature must lead to a change in the balance between dipole-dipole and scalar solid-liquid interactions, as in the case of hardwood char suspensions (Fig.4-a).

The sizes of the observed positive as well as negative DNP enhancements in char suspensions are more than an order of magnitude less than the theoretical maximum of pure scalar (+330; scalar relaxation of Abragam's Type II [8]) and pure dipolar (-330) coupling, respectively. 
One possible mechanism responsible for the lower DNP enhancement in char suspensions is the inhomogeneous molecular diffusion and magnetic relaxation processes in porous media [28].

The self-diffusion coefficients D for aqueous char suspensions were measured by PFG spin-echo NMR spectroscopy. Typical raw PFG data are shown in Fig.2. The near-linear dependence of $\ln \left(\mathrm{A}_{\mathrm{g}} / \mathrm{A}_{\mathrm{o}}\right)$ on the gradient strength $g$ was observed in fructose char suspensions. It is an important feature of the data, as it indicates that the water molecules are dominantly undergoing unbounded diffusion in this kind of chars. This observation may be caused by a hydrophobic surface character of the fructose chars. This hydrophobic character of fructose char is in good agreement with the dominant dipole-dipole (through space) type of solid-liquid hyperfine interactions, which leads to negative DNP enhancement in these char suspensions (Table 1). In contrast to fructose char suspensions the effect of restriction on the PFG-NMR diffusion measurement is clearly demonstrated in Fig. 5 by the data obtained for hardwood char suspensions. The observation suggests the hydrophilic character of the surfaces of these chars with developed porous structures. The hydrophilic character of chars is favorable to shortdistance contact hyperfine coupling at solid-liquid interface, which leads to electron delocalization from paramagnetic centers on the char surface to water protons and to positive DNP enhancement in hardwood char suspensions (Table 1).

Proton spin-lattice relaxation data generally confirm the results of molecular diffusion measurements. Analyzing the results of Table 2, one can find that in hardwood char suspensions the fraction of the water molecules in pore spaces is equal to $12.2 \%$ in contrast to only $2 \%$ in fructose chars, suggesting an essential difference in the porous structure of these chars.

Following Torrey [26], let the amount of fluid with volume $\mathrm{V}$ be bounded by the porous solid (Fig.3). The surface of solid contains a number of paramagnetic centers that are in resonance with the microwave power. We denote by $\mathrm{V}^{\mathrm{p}}$ the volume occupied by fluid molecules in pores in the immediate vicinity of these centers, and by $\mathrm{V}^{\mathrm{s}}$ (which is part of $\mathrm{V}$ ) the volume occupied by fluid molecules in the reminder of the surface layer. The nuclei in $\mathrm{V}^{\mathrm{p}}$ as well as in $\mathrm{V}^{\mathrm{s}}$ are flipped at a given rate by the external microwave power through the Overhauser effect, with characteristic relaxation times $\mathrm{T}_{1}{ }^{\mathrm{p}}$ and $\mathrm{T}_{1}{ }^{\mathrm{s}}$, respectively. Some flipped nuclei escape to the volume $\mathrm{V}^{\mathrm{o}}$ before they are relaxed, where they are exposed to the ordinary relaxation, characterized by the relaxation time $\mathrm{T}_{1}{ }^{\circ}$.

The following equation can be used [26] for experimentally observed (volumetrically average) spin-lattice relaxation time $\mathrm{T}_{1}{ }^{\text {av }}$ of the fluid and nuclear leakage factor $f$ in porous media:

$$
\begin{gathered}
\left(\mathrm{T}_{1}{ }^{\mathrm{av}}\right)^{-1}=\left(\mathrm{T}_{1}{ }^{\mathrm{o}}\right)^{-1}+\mathrm{V}^{\mathrm{s}} /\left(\mathrm{T}_{1}{ }^{\mathrm{s}}+\tau^{\mathrm{s}}\right)+\mathrm{V}^{\mathrm{p}} /\left(\mathrm{T}_{1}{ }^{\mathrm{p}}+\tau^{\mathrm{p}}\right) \\
f=\left[1+\mathrm{V}^{\mathrm{s}}\left(\mathrm{T}_{1}{ }^{\mathrm{p}}+\tau^{\mathrm{p}}\right) / \mathrm{V}^{\mathrm{p}}\left(\mathrm{T}_{1}{ }^{\mathrm{s}}+\tau^{\mathrm{s}}\right)+\left(\mathrm{T}_{1}{ }^{\mathrm{p}}+\tau^{\mathrm{p}}\right) / \mathrm{V}^{\mathrm{p}} \mathrm{T}_{1}{ }^{\mathrm{o}}\right]^{-1}
\end{gathered}
$$

where we assumed $\mathrm{V}=1 ; \tau^{\mathrm{s}}$ and $\tau^{\mathrm{p}}$ are the sticking times of molecules entering $\mathrm{V}^{\mathrm{s}}$ and $\mathrm{V}^{\mathrm{p}}$, respectively. Fig. 4 shows the dependence of the leakage factor, $f$, on the sticking times, $\tau^{\mathrm{p}}$ and $\tau^{\mathrm{s}}$, in fructose char suspensions. It follows that the $f$ value (and as a result the value of DNP enhancement) in char suspensions mainly depends on the sticking time $\tau^{\mathrm{p}}$ of water molecules in pore spaces. This "cage effect" obviously will increase with increasing pore structure tortuosity.

The main conclusions are: 1 . The main DNP processes in char suspensions occur in pore spaces of chars, and the short-range nuclear-electronic interactions in porous structures dominate the 
Semiannual Technical Report to DOE - PETC - Grant \# DE-FG22-96PC96205 9/1/96-2/28/97

DNP enhancement. Long-range interactions associated with the different kinds (nuclear and electronnuclear) of relaxation processes in the reminder of the surface layer have small or even negligible effect; 2. Synthesizing chars with substantially developed porous structures and small pore sizes, small tortuosity, and fast spin-lattice relaxation in the pore spaces leads increased DNP effects in the char suspensions.

\section{REFERENCES}

[1] B.M.Odintsov, R.L.Belford, P. J.Ceroke, and R.B.Clarkson, J. Am. Chem. Soc. 1997 (in press)

[2] B.M.Odintsov, R.L.Belford, P. J.Ceroke, and R.B.Clarkson, Surface Science, 1997 (in press)

[3] R.B.Clarkson, B.M.Odintsov, P. J.Ceroke, J.H.Ardenkjar-Larsen, M.Fruianu, and R.L.Belford, Physics

[4] R.D. Bates, Jr.; Magn. Res. Rev. 1993, 16, 237.

[5] H.C. Dorn, T.E. Glass, R. Gitti, K.H. Tsai, Appl. Magn. Res. 1991, 2, 9-27.

[6] G.G. Maresch, R.D. Kendrick, C.S. Yannoni, J. Magn. Res. 1989, 82, 41-50.

in Medicine and Biology, 1997 (in press).

[7] A. Overhauser, A., Phys. Rev. 1953, 91, 476.

[8] A. Abragam, in: Principles of Nuclear Magnetism, (Oxford Univ. Press, Oxford, 1961).

[9] R. Gitti, C. Wild, C. Tsiao, K. Zimmer, T.E. Glass, and H.C. Dorn, J.Am.Chem.Soc., 1988, 110, 2294.

[10] R.A. Wind, M.J. Duijveshijn, C. Van Der Lagt, A. Manenshijn, and J. Vriend, Prog. NMR Spectrosc. $198517,33$.

[11] R. Codrington and N. Bloembergen, J.Chem.Phys., 1958, 29, 600.

[12] J. Collingwood, and J. White, Mol.Phys., 1973, 25, 1241

[13] K.S.Rothenberger, R.F.Sprecher, S.M. Gastellano, H.L. Retcofsky, In Magnetic Resonance of Carbonaceous Solids, Botto R.B., Sanada Y., eds., Advances in Chemistry; Am. Chem. Soc., (Washington, DC, 1981), pp.581598.

[14] R.B. Clarkson, W. Wang, R.D. Brown, H.C Crookham., and R.L. Belford, Fuel, 1990, 69, 1405.

[15] H.A. Resing, Advances in Molecular Relaxation Processes, 1968, 1, pp.109-154.

[16] J.K. Thompson, J.J. Krebs, and H.A. Resing,. J.Phys.Chem. 1965, 43, 3853-3865.

[17] H.A. Resing, J.K. Thompson, and J.J. Krebs, J.Phys.Chem.1964, 68, 1621-1627.

[18] S. Kaplan, H.A. Resing. and A. Waugh, "13C NMR Chemical Shift Anisotropy for Benzene Adsorbed on Charcoal and Silica Gel” J. Chem. Phys., 59, 10, 1973, 5681-5687.

[9] E.G. Derouane, J. Fraissard, J.J. Fripiat, W.E.E.Stone. Catal. Rev., 1972, 7, 121-138. 
Semiannual Technical Report to DOE - PETC - Grant \# DE-FG22-96PC96205 9/1/96-2/28/97

[20] B.M. Odintsov, R.L. Belford and R.B. Clarkson, J.Phys. Chem. 1997, 101, 116.

[21] B. Antalek, K.Chari, Modern Phys. Letters B, 1995, 9155.

[22] C.S. Johnson, Jr., In: Encyclopedia of NMR; Grant D.M., Harris R.K., Eds.; Wiley: New York, 1996, 3, 1626.

[23] M.D.Hurlimann, K.G.Helmer, L.L.Latour, C.H.Sotak, J.Magn.Res., Ser.A, 1994, 111, 169-178.

[24] P.P.Mitra, L.L.Latour, R.L.Kleinberg, C.H.Sotak, J.Magn.Res., 1995, 114, 47-58.

[25] S. Boyer and R.B. Clarkson, Colloids Surf. A. 1994, 82, 217.

[26] H.C.Torrey, J.Korringa, D.O.Seevers, J.Ubersfeld, Phys.Rev.Letters, 1959, 3, 418.

TABLES AND FIGURES

Table 1. Experimental DNP Data in Aqueous Char Suspensions at Magnetic Field 117.5G

\begin{tabular}{|c|c|c|c|}
\hline No & Material & $\mathbf{T}_{1}^{\text {av }}, \mathbf{s}$ & $\mathbf{A}_{\max }$ \\
\hline $\mathbf{1}$ & Hardwood & $0.28 \pm 0.01$ & $+20 \pm 0.3$ \\
\hline $\mathbf{2}$ & Starch & $0.31 \pm 0.01$ & $-24 \pm 0.3$ \\
\hline $\mathbf{3}$ & Fructose & $0.49 \pm 0.02$ & $-65 \pm 2.1$ \\
\hline
\end{tabular}

Table 2. Self-Diffusion Coefficients and NMR Relaxation Data in Aqueous Char Suspensions.

\begin{tabular}{|c|c|c|c|c|c|c|c|c|c|}
\hline $\mathbf{N}$ & Material & $\begin{array}{c}\mathrm{D}^{0} * 10^{5} \\
\mathrm{~cm}_{2} / \mathrm{s}\end{array}$ & $\begin{array}{c}D^{\mathrm{S} * 10^{5}} \\
\mathrm{~cm}_{2} / \mathrm{s}\end{array}$ & $\begin{array}{l}\mathbf{V}^{0} \\
\%\end{array}$ & $\begin{array}{l}\mathbf{V}^{\mathrm{S}} \\
\%\end{array}$ & $\begin{array}{l}\mathbf{V}^{\mathbf{p}} \\
\%\end{array}$ & $\begin{array}{c}\mathrm{T}_{1}^{0} \\
\mathrm{~S}\end{array}$ & $\begin{array}{c}\mathbf{T}_{1}^{\mathrm{S}} \\
\mathbf{S}\end{array}$ & $\begin{array}{c}\mathbf{T}_{1}^{\mathbf{P}}, \\
\mathbf{S}\end{array}$ \\
\hline 1 & Hardwood & $1.6 \pm 0.2$ & $0.06 \pm 0.006$ & $6.8 \pm 0.4$ & $81 \pm 2$ & $12.2 \pm 0.6$ & $0.9 \pm 0.06$ & $0.32 \pm 0.02$ & $0.033 \pm 0.02$ \\
\hline 2 & Starch & $1.75 \pm 0.2$ & $0.1 \pm 0.01$ & $7.9 \pm 0.4$ & $89 \pm 2$ & $3.1 \pm 0.6$ & $1.02 \pm 0.06$ & $0.35 \pm 0.02$ & $0.048 \pm 0.02$ \\
\hline 3 & Fructose & \multicolumn{2}{|c|}{$1.96 \pm 0.2$} & $98 \pm 2$ & \multicolumn{2}{|c|}{$2.0 \pm 0.2$} & \multicolumn{2}{|c|}{$1.2 \pm 0.06$} & $0.04 \pm .001$ \\
\hline
\end{tabular}




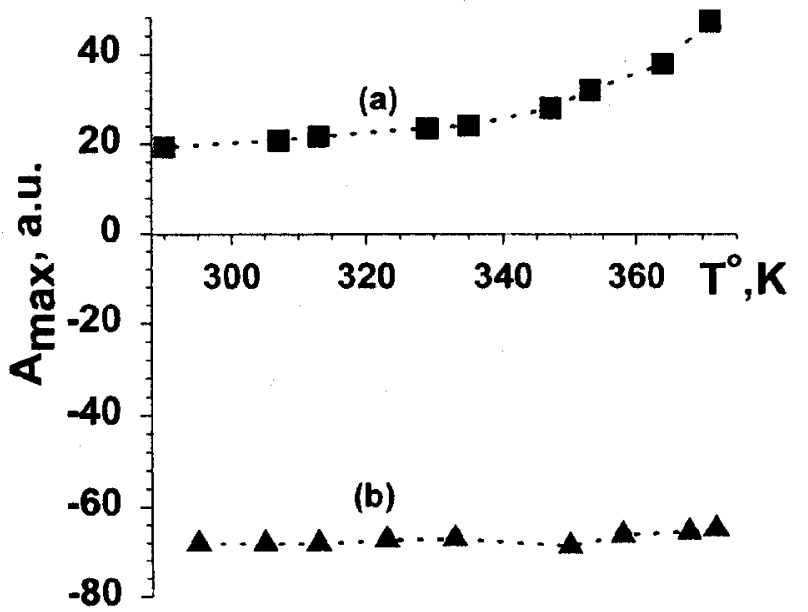

Fig. 1. Temperature dependence of DNP enhancements of water protons in aqueous suspensions of cha rs made from: a) hardwood and b) fructose. Instrument paramers: $B_{1 s}=0.1 G, \tau_{p}=0.5 \mathrm{~s}, \tau_{d}=4 \mathrm{~ms}$.

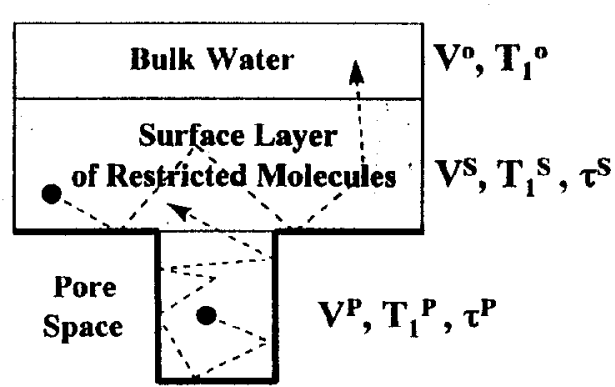

Fig. 3. Schentatic illustrating the effect of restricting boundaries on water diffusion and relaxation measurements in char suspensions.

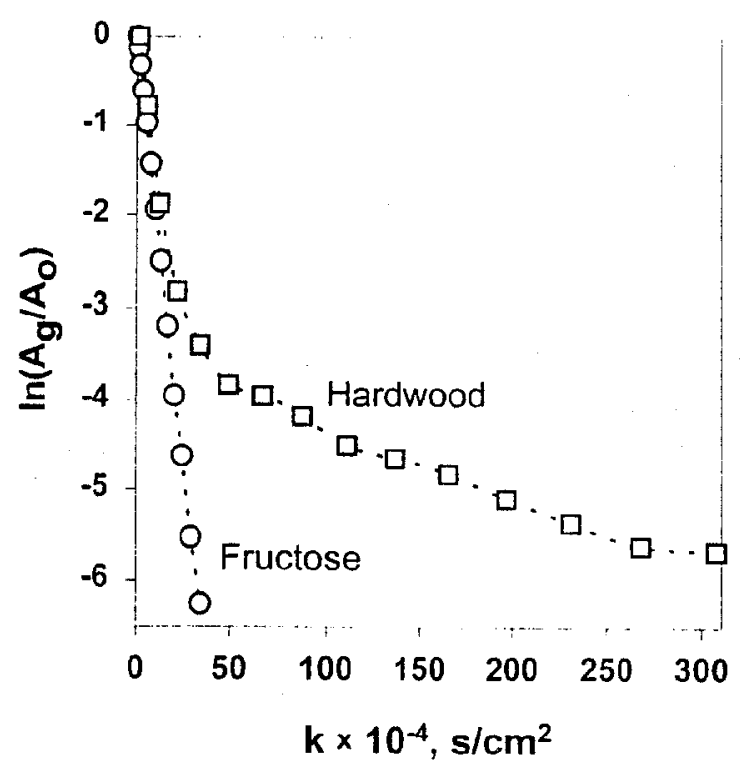

Fig. 2. Typical raw PFG-NMR data. Plot of $\ln \left(A_{r} / A_{0}\right)$ vs. $k$ for two chars saturated with water. The squares are obtained from water diffusing In hardwood char suspensions and clearly lliustrate the effects of restriction on the PFG-NMR diffusion experiment. The circles, taken from water in fructoae suspensions under the same experimental conditions, represent the curve for unrestricted diffusion.

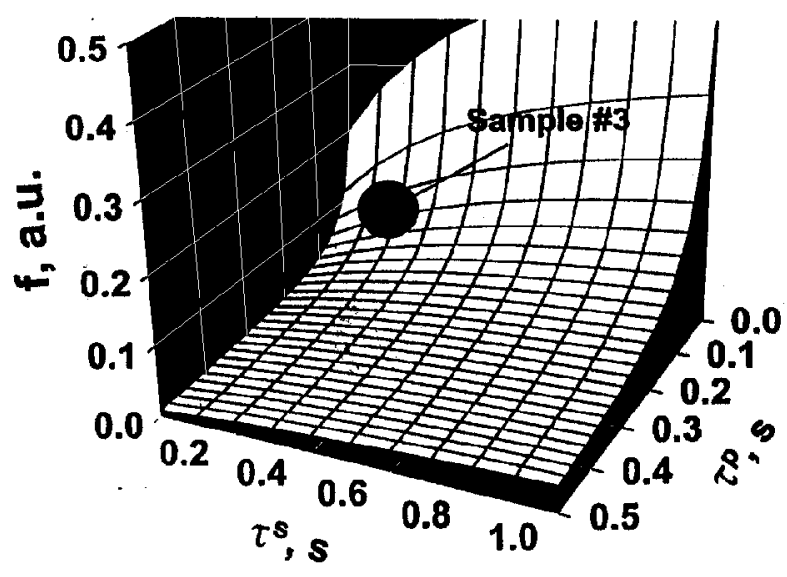

Fig. 4. The dependence of leaknge factor $f$ on the sticking times of $t^{\prime}$ and $\tau^{*}$ for fructose clonr suspensions. 